\title{
ZIEGLER-NICHOLS PID CONTROLLER FOR EFFECTIVE PAY-LOAD TORQUE RESPONSES AND TIP-VIBRATIONS OF DOUBLE LINK MANIPULATOR
}

\author{
Lelin Kumar. ${ }^{1}$, Balasubbareddy. ${ }^{2}$, Madhu. ${ }^{3}$ \\ ${ }^{1}$ M.Tech Research Scholar, ${ }^{2}$ Professor, ${ }^{3}$ Assistant Professor, Department of Electrical \& Electronics Engineering, \\ Prakasam Engineering College, Kandukur (A.P), India
}

\begin{abstract}
Robotic Technology is an imitation for human being's. It is an electro mechanical modelling objects are important aspect of manipulator. A Manipulator is a machine able to drive the robot. This paper describes and investigates on effective pay-load torque responses, tip-vibrations. This paper presents modelling and simulation of the double link manipulator using the proposed PID Controller. Here the proposed Ziegler-Nichols proportional-integral-derivative controller (PID controller) initiates more advantageous in the view of better performance and flexible operation of manipulator. First, the electro mechanical object was modeled and simulated using State space technique. Here the torque responses and end tip vibrations are assigned by state space variables. The entire two link manipulator topology was investigated and modeled, simulated. The proposed control strategy carries a back-back feed forward controller can be used for flexible operation of manipulator. Here, the simulation was done by using M-File Technique in Control tool box of MATLAB.
\end{abstract}

Keywords: Double Link Manipulator, Ziegler-Nichols PID Controller, Pay-Load Torque Responses, Tip-Vibrations, State space technique, back-back feed forward controller

\section{INTRODUCTION}

The robot technology is advancing rapidly. The industry is moving from the current state of automation to robotization, to increase productivity and to deliver uniform quality. Robots like and robot like manipulators is now commonly employed in hostile environment, such as at various places in an atomic plant for handling radioactive materials. Robots are being employed to construct and repair space stations and satellites. There are now increasing number of applications of robots such as in nursing and aiding a patient .Micro robots are being designed to do damage control inside human veins. Robots like systems are now employed in heavy earth-moving equipment. It is not possible to put up an exhaustive list of robot applications. One type of robot commonly used in the industry is a robotic manipulator or simply a manipulator or a robotic arm. A Manipulator is a machine that has functions similar to human upper limbs, and moves the objects spatially. The Robotics Industries Association (RIA) of USA defines the robot as "A reprogrammable, multifunctional manipulator designed to move material through variable programmed motions for the performance of a variety of tasks".

Review of the research work reveals that much work has been done on various aspects of control of manipulators using PID controllers, nonlinear PD controllers, different algorithms etc., but the results are not generalized fit well to everyone. Thambirajah Ravichandran, David Wang, Glenn Heppler et al studied on numerical optimization techniques for simultaneously optimizing design parameters of a 2-link rigid manipulator and a nonlinear gain PD controller designed for performing multiple tasks. They cannot make it to study the vector optimization for generating the entire set of optimal solutions to simultaneous design optimization methodology.

Reza Fotouhi-C, Walerian Szyszkowski, Pete N. Nikiforuk, et al studied on two phase trajectory planning for a two link rigid manipulator using two stages. Geometric trajectory planning and speed control using a nonlinear scaling of time variable. It was demonstrated that specified velocity is to be followed. J.W.S. Chong, S.K. Ong, A.Y.C. Nee, K. Youcef-Youmi, et al studied the use of an augmented reality(AR) environment for facilitating intuitive robot programming, and presents a methodology for planning collision-free paths for an n-d.o.f. (degree-of-freedom) manipulator in a 3D AR environment. The methodology is interactive because the human is involved in defining the free space or collision-free volume (CFV), and selecting the start and goal configurations. Anto'nio Lopes, Fernando Almeida et al studied on position controlled commercial industrial robot. Combination of the two manipulators behaves as a single manipulator having the impedance and force control dynamic performance of the RCID, as well as the workspace and trajectory tracking 
bandwidth of the industrial robot. Force-impedance control of the RCID, and experimental results on typical tasks that involve end-effectors contact with uncertain environments of unknown stiffness are presented. Alessandro Gasparetto, Vanni Zanotto et al studied on a technique for optimal trajectory planning of robot manipulators. The proposed technique enables one to take into account kinematics constraints on the robot motion, expressed as upper bounds on the absolute values of velocity, acceleration and jerk. The algorithm has been tested in simulation yielding good results, also in comparison with those provided by another important trajectory planning technique. Changhoon Kim, Jae H. Chung, Daehie Hong et al studied on robust feedback linearization utilization to minimize the undesirable effect of external disturbances such as static and Coulomb friction and nonlinear compliance of the pneumatic cylinder stemming from the compressibility of air. The developed coordination control method demonstrated its efficacy in terms of deburring accuracy and speed. Karim Abdel-Malek, Jingzhou Yang et al studied on algorithm and accompanying experimental code is aimed at calculating the volume swept by an object held by the end-effector of a robotic manipulator. A general formulation has been presented that addresses this motion that allows the visualization of the volume which the robot has swept.

\section{OVERVIEW OF DOUBLE LINK}

\section{MANIPULATOR}

To perform an assigned task or to attain a desired position, a manipulator is required to accelerate from rest, travel at specified path, and finally decelerate to stop. To accomplish this, trajectory, controlling torques is applied by the actuators at the manipulator joints. This torques is computed from the equations of motion of the manipulator, which describe the dynamics of the manipulator. The dynamic model is very useful for mechanical design of the structure, choice of actuator, computer simulation of performance, determination of control strategies, and design of control system. The dynamic model and generated trajectory constitute the inputs to the motion-control system of the manipulator. The problem of manipulator control is to find the time behavior of the forces and torques delivered by the actuators for executing the assigned task. Both the manipulator motion control and its force interaction with the environment are monitored by the control algorithm. The above exposed problems will lead to the study of control systems for manipulator and several techniques.

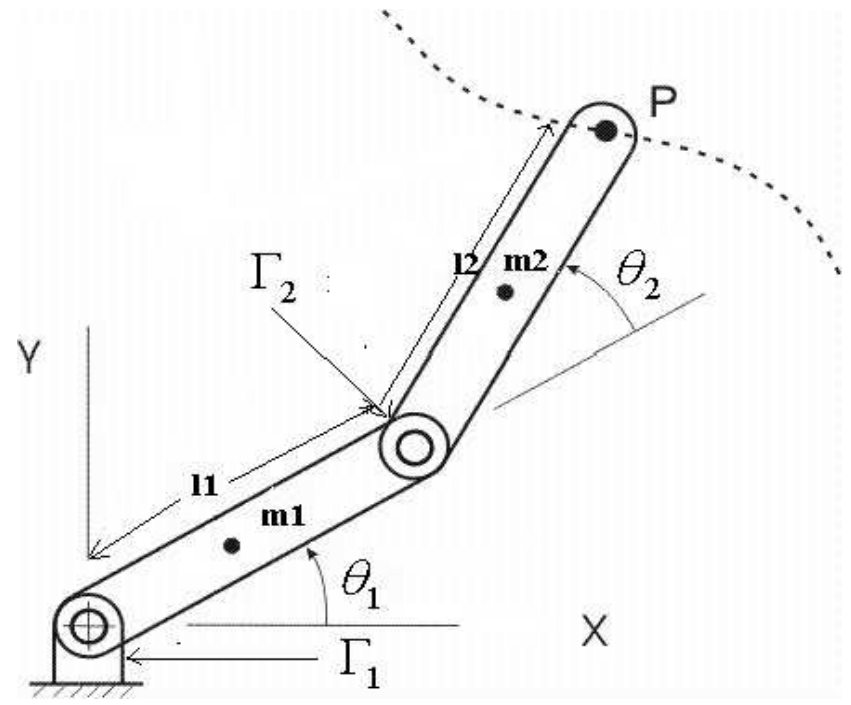

Figure.1.Double link rigid manipulator

The tasks to be performed by the manipulator are (1) to move the end-effector along a desired trajectory, and (2) to extract a force on the environment to carry out the desired task. The controller of manipulator has to control both tasks, the former is called position control (or trajectory control) and the latter force control. A schematic sketch of a typical controller is shown below.

The positions, velocities, forces, torques are measured by sensors and based on these measurements and the desired behavior, the controller determines the inputs to the actuators on the robot so that the end effector carries out the desired task as closely possible.

\section{PROPOSED CONTROLLER FOR}

\section{MANIPULATOR}

A Ziegler-Nichols proportional-integral-derivative controller (PID controller) is a generic control loop feedback mechanism widely used in industrial control systems. A PID controller attempts to correct the error between a measured process variable and a desired set point by calculating and then outputting a corrective action that can adjust the process accordingly. 


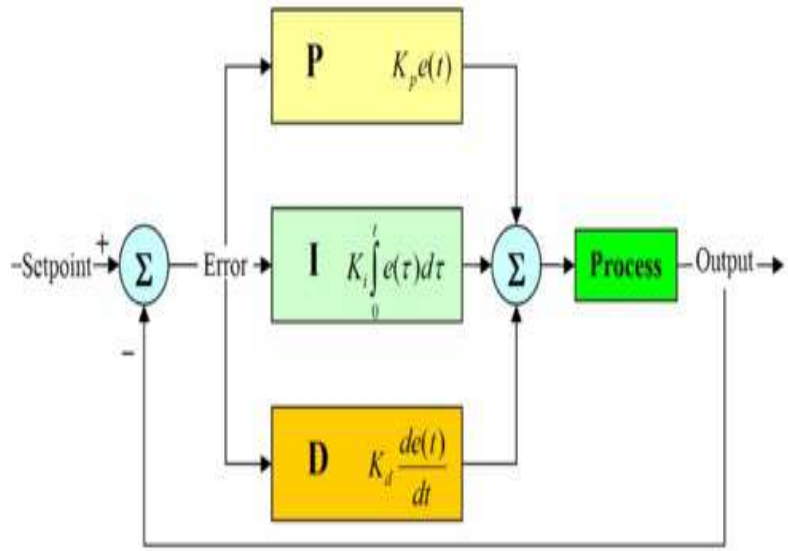

Figure.2. Block diagram of Ziegler-Nichols PID controller

The PID controller calculation (algorithm) involves three separate parameters; the Proportional, the Integral and Derivative values. The Proportional value determines the reaction to the current error, the Integral determines the reaction based on the sum of recent errors and the Derivative determines the reaction to the rate at which the error has been changing. The weighted sum of these three actions is used to adjust the process via a control element such as the position of a control valve or the power supply of a heating element. By "tuning" the three constants in the PID controller algorithm the PID can provide control action designed for specific process requirements.

The response of the controller can be described in terms of the responsiveness of the controller to an error, the degree to which the controller overshoots the set point and the degree of system oscillation. Note that the use of the PID algorithm for control does not guarantee optimal control of the system or system stability. Some applications may require using only one or two modes to provide the appropriate system control. This is achieved by setting the gain of undesired control outputs to zero. A PID controller will be called a PI, PD, P or I controller in the absence of the respective control actions. PI controllers are particularly common, since derivative action is very sensitive to measurement noise, and the absence of an integral value may prevent the system from reaching its target value due to the control action.

\subsection{Ziegler-Nichols PID Tuning}

If the PID controller parameters (the gains of the proportional, integral and derivative terms) are chosen incorrectly, the controlled process input can be unstable, i.e. its output diverges, with or without oscillation, and is limited only by saturation or mechanical breakage. Tuning a control loop is the adjustment of its control parameters (gain/proportional band, integral gain/reset, derivative gain/rate) to the optimum values for the desired control response. The optimum behavior on a process change or set point change varies depending on the application. Some processes must not allow an overshoot of the process variable beyond the set point if, for example, this would be unsafe. Other processes must minimize the energy expended in reaching a new set point. Generally, stability of response (the reverse of instability) is required and the process must not oscillate for any combination of process conditions and set points.

Table 1 Comparison of Different Controllers

\begin{tabular}{|c|c|c|c|}
\hline S.No & Method & Advantages & Disadvantages \\
\hline 1 & $\begin{array}{l}\text { Manual } \\
\text { Tuning }\end{array}$ & less & more \\
\hline 2 & $\begin{array}{l}\text { Software } \\
\text { Tools }\end{array}$ & medium & more \\
\hline 3 & $\begin{array}{l}\text { Cohen- } \\
\text { Coon }\end{array}$ & more & more \\
\hline 4 & $\begin{array}{l}\text { Ziegler- } \\
\text { Nichols }\end{array}$ & more & Less \\
\hline
\end{tabular}

Some processes have a degree of non-linearity and so parameters that work well at full-load conditions don't work when the process is starting up from no-load. This section describes some traditional manual methods for loop tuning. There are several methods for tuning a PID loop. The most effective methods generally involve the development of some form of process model, and then choosing P, I, and D based on the dynamic model parameters. Manual tuning methods can be relatively inefficient. The choice of method will depend largely on whether or not the loop can be taken "offline" for tuning, and the response time of the system. If the system can be taken offline, the best tuning method often involves subjecting the system to a step change in input, measuring the output as a function of time, and using this response to determine the control parameters.

\subsection{Proposed Control Algorithm}

The basic ziegler-nichols PID algorithm presents some challenges in control applications that have been addressed by minor modifications to the ziegler-nichols PID form. One common problem resulting from the ideal ziegler-nichols PID implementations is integral windup. This can be addressed by:

1. Initializing the controller integral to a desired value

2. Disabling the integral function until the PV has entered the controllable region

3. Limiting the time period over which the integral error is calculated

4. Preventing the integral term from accumulating above or below pre-determined bounds

Many ziegler-nichols PID loops control a mechanical device (for example, a valve). Mechanical maintenance can be a major cost and wear leads to control degradation in the form 
of either station or a dead band in the mechanical response to an input signal. The rate of mechanical wear is mainly a function of how often a device is activated to make a change. Where wear is a significant concern, the ziegler-nichols PID loop may have an output dead band to reduce the frequency of activation of the output (valve). This is accomplished by modifying the controller to hold its output steady if the change would be small (within the defined dead band range). The calculated output must leave the dead band before the actual output will change.

The proportional and derivative terms can produce excessive movement in the output when a system is subjected to an instantaneous "step" increase in the error, such as a large set point change. In the case of the derivative term, this is due to taking the derivative of the error, which is very large in the case of an instantaneous step change. As a result, some ziegler-nichols PID algorithms incorporate the following modifications. The Derivative of output In this case the ziegler-nichols PID controller measures the derivative of the output quantity, rather than the derivative of the error. The output is always continuous (i.e., never has a step change). For this to be effective, the derivative of the output must have the same sign as the derivative of the error and Set point ramping In this modification, the set point is gradually moved from its old value to a newly specified value using a linear or first order differential ramp function.

This avoids the discontinuity present in a simple step change. The Set point weighting Set point weighting uses different multipliers for the error depending on which element of the controller it is used in. The error in the integral term must be the true control error to avoid steady-state control errors. This affects the controller's set point response. These parameters do not affect the response to load disturbances and measurement noise.

\section{DOUBLE LINK MANIPULATOR TEST SYSTEM WITH ZIEGLER-NICHOLS PID CONTROLLER}

The test System of double link manipulator was modeled and simulated in MAT Lab/Simulink Control tool box. Here the electro mechanical model was initiated by using state space representation and the each aspect of proposed test system assigned as a state variables. Figure. 3 Represents the modeling and simulation of double link manipulator with a proposed controller

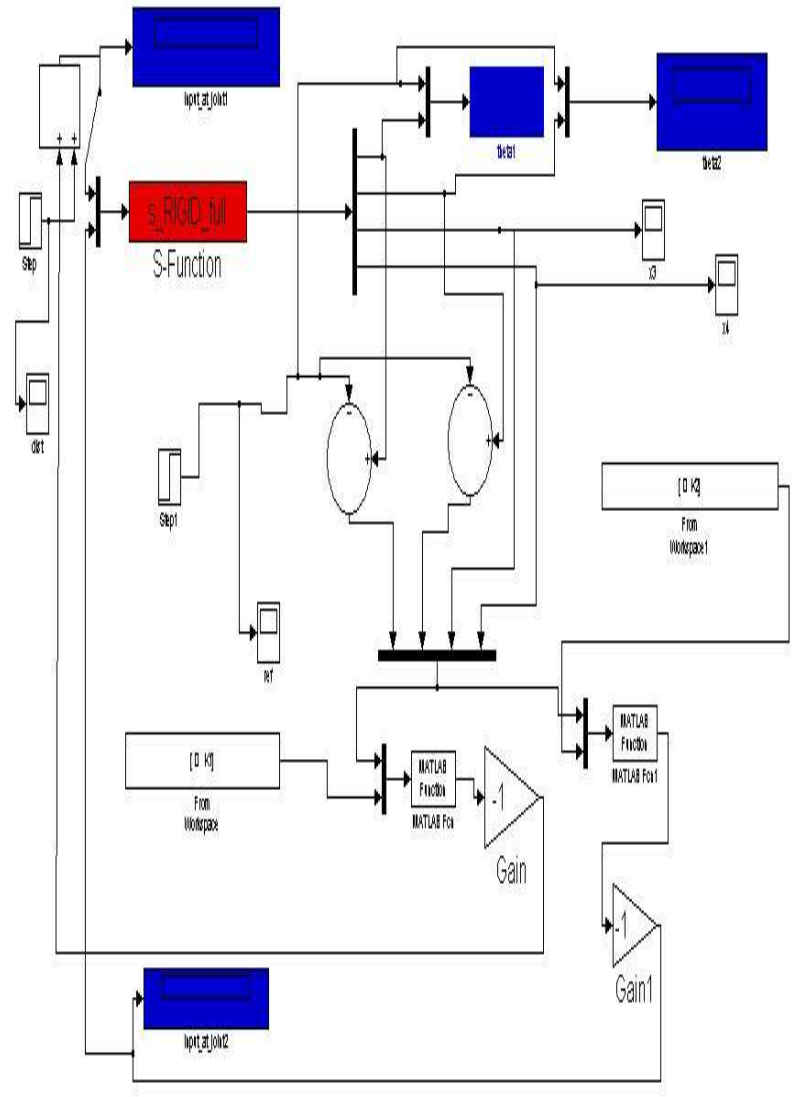

Figure.3 Simulating Diagram for Double Link Manipulator with proposed Controller

\section{RESULTS AND DISCURSIONS}

The double link manipulator with a proposed controller gives a responses of two parameters like pay load torque response and tip vibrations etc. Here the figure. 4 and figure. 5 represents two responses of two links of manipulator i.e. Theta_1 and Theta_2 respectively.

Theta_1 response consists on $\mathrm{x}$-axis as a pay load torque response and on $y$-axis tip vibrations response and, for Theta_2 posses the same for simplicity. Here from figure.4 and 5 initiates the responses of torque and tip vibrations etc. Apart from that, the above two parameters describes the flexibility of manipulator. The entire double link was controlled by using proposed PID Controller for Flexibility which gives two responses for two links of manipulator, in that each was represented as a Theta_1 and Theta_2 respectively. Overall, the two responses case some flexibility due to proposed PID Controller itself. 


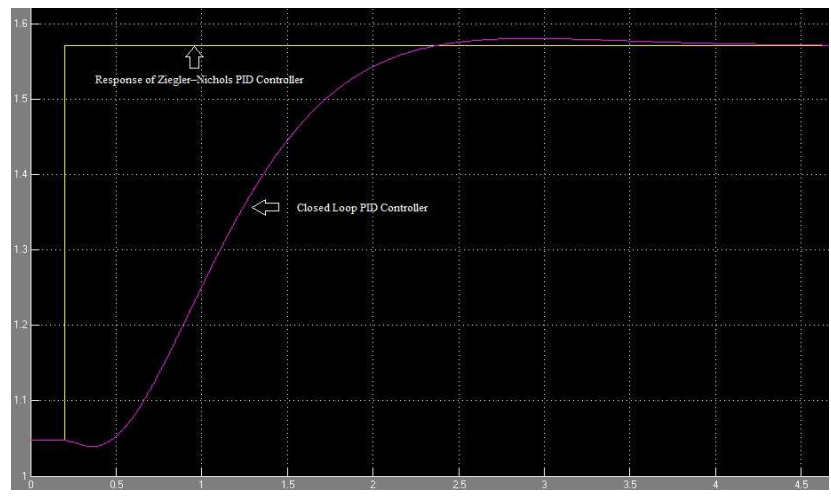

Figure4. Comparison Responses of Theta_1 of Double link Manipulator

The figure.4 represents a Theta_1 response of double manipulator with a closed loop PID Controller and proposed Ziegler-Nichols PID Controller itself. From figure.4, on xaxis pay load torque of manipulator was represented and on $y-$ axis tip vibrations are represented. Finally That_1 response initiates comparison of proposed PID Controller with a normal closed loop PID Controller. Out of these two, the proposed Ziegler-Nichols PID Controller will show a better $4-5 \%$ of working performance.

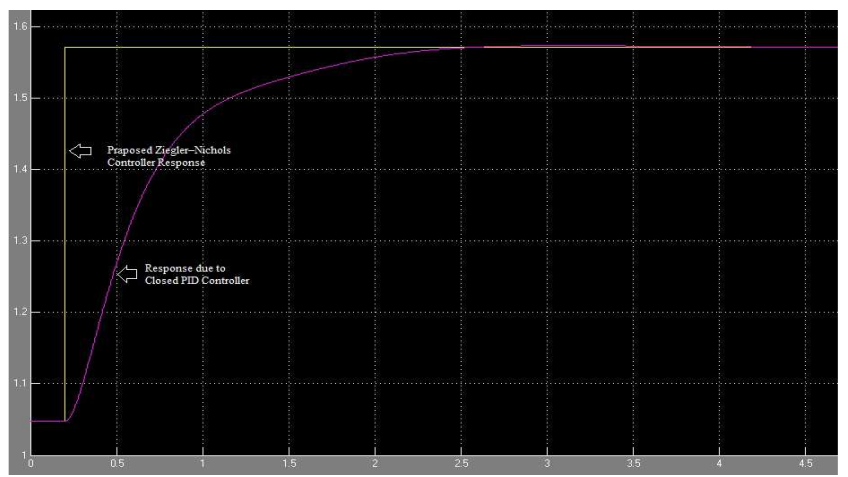

Figure5 Comparison Responses of Theta_2 of Double link Manipulator

For Theta_2 also it follows the same. Similarly, it shows a 5$8 \%$ of flexible performance in case of Theta_2 response. So, the proposed PID controller initiates better performance charectestics for theta_1 and theta_2 justified as a flexible operation of the double link manipulator at far end.

\section{CONCLUSIONS}

In this paper, we describe the two link responses of double link manipulator at far end like Theta_1 and Theta_2 respectively. The two important aspects of double link manipulator are pay-load torque and tip-vibrations are investigated. The proposed Ziegler-Nichols PID Controller showing a better performance than a normal Closed Loop controlled tuning. The performance charectestics of theta_1 and theta_2 are inverses form $2-3 \%$ to $5-6 \%$. The entire double link manipulator was simulated by using M-File technique in Control tool box of MAT Lab. The Overall topology was studied and modeled, simulated. We concluded for the flexible operation of double link manipulator, we propose a Ziegler-Nichols PID Controller show a robust and better performance.

\section{ACKNOWLEDGEMENTS}

The author wishes to thank Prof.M.Bala Subbareddy and Madhu.T and the faculty of Department of Electrical \& Electronics Engineering, for their contribution in this project.

\section{REFERENCES}

[1]. Thambirajah Ravichandran, David Wang, Glenn Heppler (2006), "Simultaneous plant-controller design optimization of a two-link planar manipulator", Journal of Mechatronics, Volume 16, Pages: 233-242

[2]. Reza Fotouhi-C, Walerian Szyszkowski, Peter N. Nikiforuk (2002), "Trajectory planning speed control for a two-link rigid manipulator", Journal of mechanical design, Volume 124,Pages: 585-589.

[3]. Fotouhi-C., R., Nikiforuk, P., and Szyszkowski, W., (1998), "Combined trajectory planning and parameter identification of a two link rigid manipulator", Proceedings of ASME Design Engineering Technical conferences, Atlanta, Georgia.

[4]. Fotouhi-C, R., Szyszkowski, W., Nikiforuk, P. N., and Gupta, M.M., (2000), "Parameter identification and trajectory following of a two link rigid manipulator", Journal of Systems and Control Engineering, proceedings part I, 213.

[5]. J.W.S. Chong, S.K. Ong, A.Y.C. Nee, K. YoucefYoumi, "Robot programming using augmented reality: An interactive method for planning collision free paths." Journal of Robotics and Computer-Integrated manufacturing.

[6]. António Lopes, Fernando Almeida (2008), “A force impedance controlled industrial robot using an active robotic auxiliary device", Journal of Robotics and Computer - Integrated manufacturing, Volume 24, Pages: 299-309.

[7]. Karim Abdel-Malek, Jingzhou Yang (2005), "Sweeping of an object held by a robotic endeffector", Journal of Robotics and Computer Integrated manufacturing, Volume 21, Pages: 159-173.

[8]. Denny Oetomo, Marcelo H. Ang Jr, (2007) "Singularity robust algorithm in serial manipulators", Journal of Robotics and Computer Integrated manufacturing

[9]. Alessandro Gasparetto, Vanni Zanotto (2008), “A technique for time-jerk optimal planning of robot 
trajectories", Journal of Robotics and Computer Integrated manufacturing, Volume 24, Pages: 415-426.

[10]. K.A.Atia and M.P.Cartmell, (1999) "A general dynamic model for a large scale 2-DOF Planar parallel manipulator," Robotica, 17(6), 675-684.

[11]. Z.Shiller and H.H.Lu,(1998), " Computation of path constrained time optimal control motions with dynamic singularities," Transaction of ASME Journal of Dynamic Systems, Measurement and Control,114(2), 34-40.

\section{BIOGRAPHIES}

Mr.V.Lelin Kumar received B.Tech in Electrical Engineering from JNT University, Kakinada. He Working as M.Tech Research Scholar in Power Electronics from Prakasam Engineering College, Kandkur under JNTU Kakinada. His areas of interest include power electronics and FACTS, Control of AC\& DC Drives

Mr. M. Balasubbareddy, HOD of EEE Department in Prakasam Engineering College pursuing Ph.D from JNTU Kakinada $\mathrm{He}$ got M.Tech in the specialization of Power Systems from NIT, Trichy. He completed B.Tech from Madras University in the specialization of Electrical Engineering. He published many engineering Letters and Peer-Journal publication at national and international level. His area of research include power electronics, control of AC\&DC Drives, special machines etc.

Mr.T.Madhu received M.Tech in Power Electronics from JNT University, Kakinada. He received B.Tech in Electrical Engineering from JNT University, Kakinada. He is Assistant Professor of Electrical Engineering Department at Prakasam Engineering College, Kandkur (A.P), and India. He published various research articles and letters on Power Systems Engineering. His areas of interest include power electronics and FACTS, Control of AC\& DC Drives. 